\title{
An Innovative Probabilistic Methodology for Net Transfer Capacity Evaluation
}

\author{
F. Adinolfi, S. Massucco \\ M. Saviozzi, F. Silvestro \\ University of Genova - DITEN \\ francesco.adinolfi@unige.it, stefano.massucco@unige.it, \\ federico.silvestro@unige.it, matteo.saviozzi@edu.unige.it
}

\author{
E. Ciapessoni, D. Cirio \\ A. Pitto \\ RSE - Ricerca sul Sistema Energetico \\ Milan, Italy \\ diego.cirio@rse-web.it, andrea.pitto@ rse-web.it
}

\begin{abstract}
In the context of transmission system planning, research proposes methods to assess the effect of uncertainties of power system operating condition due to forecasting errors of intermittent generation and loads. In particular probabilistic power flow methods are illustrated to calculate the probability distributions of the voltages and the branch currents, starting from the distributions of power injections/absorptions. These uncertainties play a key role in the operational planning of power systems, as certain configurations of load and intermittent generation can cause security problems.

This paper aims to propose a probabilistic methodology to assess Net Transfer Capacity (NTC) among network areas, which quantifies forecast error uncertainties by applying the Point Estimate Method (PEM) combined with Third Order Polynomial Normal (TPN) Transformation. This approach is compared with a conventional NTC assessment technique and has been validated against Monte-Carlo benchmark on an IEEE test system.
\end{abstract}

Index Terms-Net Transfer Capacity, Total Transfer Capacity, Probabilistic Security Assessment, Point Estimate Method

\section{INTRODUCTION}

The increasing uncertainties in power systems originated by liberalized market and the penetration of Renewable Energy Resources (RES) raise more and more attention to security issues [1] - [4]. RES forecast uncertainties are affected by several factors, e.g. prediction techniques, RES aggregation level and forecast horizon. Moreover, correlations in forecast errors are likely to occur, especially as far as RES generation and loads are weather dependent. Correlation in forecast errors of power injections could emphasize the deviation of the operating condition with respect to the forecast state, possibly exacerbating security issues [5], [6].

Power system security assessment techniques can strongly benefit from probabilistic approaches, as these can efficiently quantify the effect of uncertainties on the operating condition. This work provides a novel probabilistic procedure to calculate the Net Transfer Capacity (NTC) among areas accounting for RES and load forecast uncertainties. The proposed method is based on coupling the Point Estimate Method (PEM) scheme with Third order Polynomial Normal (TPN) Transformation, which represents the major original contribution in the context of NTC studies.

The rest of the paper is organized as follows: Section II introduces some definitions and the conventional NTC calculation procedure. Section III presents the probabilistic NTC methodology accounting for RES and load forecast error uncertainties. Section IV describes the test system and the simulation scenarios, and it reports and discusses the results. Section V concludes, while Appendix describes PEM and TPN Transformation in detail.

\section{Net and Total Transfer CAPACity: DEFINitions AND CONVENTIONAL ASSESSMENT TECHNIQUE}

After briefly recalling some definitions, the section describes the basic procedure for Total Transfer Capacity (TTC) evaluation and the conventional method to calculate the NTC.

\section{A. Transfer Capacity definitions}

The definition of transmission capacities is given by European network of Transmission System Operators for Electricity (ENTSO-E) in [7] and it is briefly recalled here:

- Total Transfer Capacity (TTC) is the maximum exchange programme between two areas compatible with operational security standards, applicable to each system if future network conditions, generation and load patterns were perfectly known in advance.

- Transmission Reliability Margin (TRM) is a security margin that copes with uncertainties on the computed TTC values.

- Net Transfer Capacity (NTC) is the maximum exchange programme between two areas compatible with security standards applicable in both areas and taking into account the technical uncertainties on future network conditions. NTC is evaluated according to the following formula:

$$
N T C=T T C-T R M
$$

TTC is set by physical realities that may prevent operation of the system according to security rules [8]: (a) current limits; (b) voltage limits; (c) stability limits (due to frequency, voltage and angle instability). These limits must be respected also in post-contingency conditions, according to the operating security criteria (typically $N-1$ criterion).

TTC evaluation is mostly influenced by: (1) the capability of the single connections of the considered network corridor; (2) The geographic location of the generators and loads with respect to the above mentioned corridor. 


\section{B. Basic algorithm for TTC Evaluation and Conventional NTC Assessment}

The procedure exploited in this work to evaluate the TTC is taken from [8] and it is based on the definition of a reference scenario. This scenario can be determined by optimal power flow technique or can be defined by the Transmission System Operator in order to study a real case.

Let $A$ and $B$ the two areas under investigation for TTC evaluation. Starting from a reference scenario, the generated power is shifted from one area to the other in order to cause additional cross-border flows. This is done by increasing the generation in $A$ step by step and decreasing the generation in $B$ by the same steps, thus obtained a new grid operating state. The modification of the generator set-points doesn't involve renewable sources, but only the dispatchable units. The consumer loads in both areas remain unchanged. The generation stress is performed until one of the following conditions occurs in the new operating state:

- the generation variation in one area cannot be compensated by the generation in the second area due to the attainment of generators minimum or maximum active power limits

- static security violations in $N$ condition

- static security violations during $N-1$ analysis applied to all branches

A dichotomy algorithm is used to determine the maximum stress which does not cause any security violations. Dynamic security assessment is not included in the TTC calculation procedure, up to now. Some dynamic simulations may be run in the final operating point, to check the fulfillment of dynamic security requirements.

The conventional procedure for the NTC evaluation, implemented in this work, is based on equation (1): after the deterministic TTC evaluation a TRM value, equal to the $5 \%$ of TTC, is applied.

\section{PRoposed PROBABILISTIC APPROACH FOR NTC ASSESSMENT}

This section discusses the probabilistic models needed to characterize the RES and load forecast errors, and proposes the probabilistic method which assesses the NTC among grid areas, accounting for the above mentioned uncertainties.

\section{A. Probabilistic modeling of the forecast errors}

Statistical dependences among the forecast errors may increase problem complexity. To this aim, two aspects are analyzed in detail: (1) the marginal distributions of the forecast errors of RES and loads; (2) possible dependences among the forecast errors. As for point 1, the marginal distributions for the $k$-hour ahead forecast errors of RES (solar and wind plants) can be derived from climatological models or from more advanced models like ensemble forecasts [4]. The approach presented in the simulations implements models drawn from different studies [3]. In particular, the non-symmetry of the forecast errors, derived from statistical analysis of historical data, suggests the use of non-symmetric distributions (like beta distributions) for wind and solar generation forecast errors. It is worth remarking that systematic errors in forecasts may determine a non-null mean value for the forecast errors, especially with regard to RES generation.

The standard deviation of RES generation forecast errors depends on [9] - [10]:

- Level of aggregation of RES: the larger is the number of wind/solar farms aggregated into the same equivalent generation, the lower is the standard deviation expressed in $\%$ of the total rating;

- Geographic extension of the RES aggregation: given the same number of aggregated RES sources, the larger the area where they are distributed the higher the compensation effect among RES, thus the smaller is the standard deviation again expressed in \% of the total rating;

- The forecast time horizon: typically the larger the time horizon the larger the variance in forecast errors.

Other factors (like the day of the year or the sky clearness) affect the standard deviation of the forecast errors on specific RES typologies (solar farms) and they are taken into account too [10]. The standard deviation associated with load forecasts is usually very low (typically 1-4 \% of the rated power).

As for point 2, different techniques, like the Nataf transformation [11] and the TPN Transformation [12], have been proposed in literature to simulate the behavior of dependent stochastic variables without using time consuming MonteCarlo techniques. The present work adopts the TPN Transformation to model input dependencies, as the input distributions may significantly differ from the normal distribution: in fact, this transformation allows to pass from dependent not normal variables to independent (possibly normal) variables.

\section{B. The proposed NTC calculation method}

The conventional NTC evaluation proposed in the previous section seems pragmatic; however, the reliability margin does not explicitly depend on the $k$-hour ahead RES and load forecast errors referring to the scenarios studied in operational planning sessions. The proposed probabilistic evaluation (summarized in Figure 1)intends to better evaluate the NTC, taking into account the RES and load forecasting errors.

The proposed methodology is based on two main points:

1) Calculation of TTC Probability Density Function (PDF), resulting from the uncertainties on the RES/loads forecasting errors.

2) The probabilistic NTC is derived starting from the Cumulative Distribution Function (CDF) of TTC. In particular, the NTC is defined as the TTC value having a probability of being exceeded equal to $\alpha: N T C_{\alpha}=$ $C D F_{T T C}^{-1}(1-\alpha)$, where $C D F_{T T C}$ is the cumulative distribution function related to the TTC evaluated at point 1. Parameter $\alpha$ can be evaluated by each TSO based on its yearly targets for security requirements: in fact $\alpha=\frac{H}{8760}$ where $H$ indicates the number of hours when total transfer capacity is higher than the $1-\alpha$ quantile. 


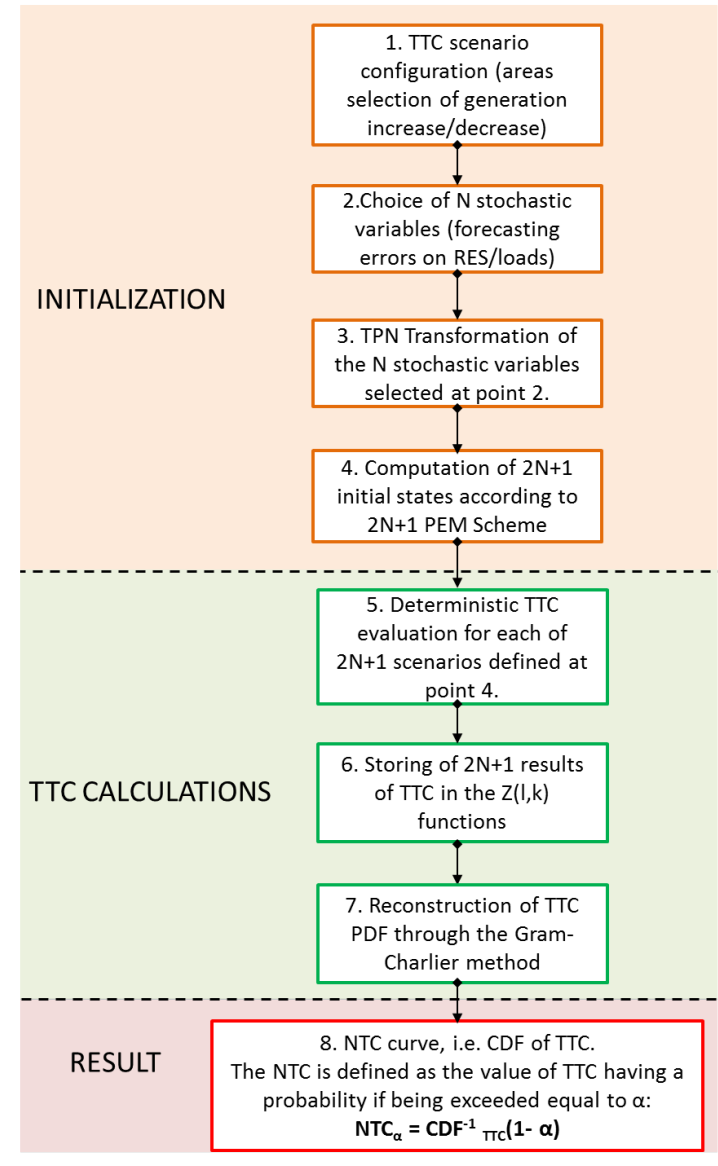

Fig. 1. Probabilistic NTC evaluation procedure

In order to execute point 1 the Point Estimate Method (PEM) [13] - [12] is implemented in the security assessment platform described in [14]. This technique requires to compute a finite set of initial power system states characterized by different combinations of values for the stochastic inputs, and to apply the TTC algorithm to this set of states. In particular, in this work the $2 N+1$ PEM scheme, where $N$ represents the number of stochastic inputs, is exploited. This scheme requires the application of the algorithm aimed at TTC evaluation on $2 N+1$ different initial power system states. In addition the $2 N+1$ PEM represents a suitable tradeoff between accuracy and computational burden. $2 N+1$ PEM technique provides good results with independent stochastic inputs, while TPN Transformation is used to account for input dependence. The fundamentals of $2 N+1$ scheme and of the TPN Transformation are presented in the Appendix.

\section{TEST SYSTEM AND SIMULATION RESUltS}

The proposed algorithm for the NTC evaluation has been tested on IEEE RTS-96 network [15]. Its one line diagram is reported in Figure 2. This network is divided into 3 macroareas: in turn, each macroarea has two areas at different voltage levels, respectively $138 \mathrm{kV}$ and $230 \mathrm{kV}$. All $230 \mathrm{kV}$ areas are labeled with even numbers, while all $138 \mathrm{kV}$ areas are labeled with odd numbers. For simulation purposes, six conventional units have been replaced with three wind farm injections and three solar plant injections (shown in Figure 2) with equivalent ratings and active power set-points so that the original loadflow is not altered. The loads and the generators set-points have been reduced by a factor equal to $2 / 3$ with respect to the RTS-96 configuration in [15].

\section{A. Conventional calculation of NTC}

Four simulation scenarios are shown in Table I together with the TTC and NTC values calculated via the conventional method. Columns "Area UP" and "Area Down" identify respectively the generation increase area and the generation decrease area. The tolerance between the last secure and the first insecure generation increment in the dichotomy algorithm is set to $2 \mathrm{MW}$. The TRM value is set to $5 \%$ of the corresponding TTC value.

TABLE I

SiMULATION SCENARIOS

\begin{tabular}{rrrrrr}
\hline ID & $\begin{array}{r}\text { Area } \\
\text { UP }\end{array}$ & $\begin{array}{r}\text { Area } \\
\text { DOWN }\end{array}$ & $\begin{array}{r}\text { TTC } \\
\text { [MW] }\end{array}$ & $\begin{array}{r}\text { NTC } \\
\text { [MW] }\end{array}$ & Stop \\
\hline A & 4 & 6 & 366 & 348 & N-1 analysis \\
B & $3-4$ & $1-2$ & 762 & 724 & N-1 analysis \\
\hline
\end{tabular}

In particular, the TTC for scenario A is limited by the overload of branch between nodes 223 and 318 due to the outage of branch 121-325; the TTC calculated for scenario $\mathrm{B}$ is constrained by the overload of branch connecting nodes 113 and 215 due to the outage of branch 123-217.

\section{B. Probabilistic calculation of NTC}

As far as probabilistic modeling is concerned, Beta distributions are used to model forecast errors of solar and wind plants. Realistic values of standard deviations equal to $15 \%$ and of $25 \%$ (for a 24 hour time horizon of forecast) are derived from literature [9], [10] to model errors on wind turbine (WT) and solar panel (SP).

The validation process consists into two steps: (I) comparing the result of the proposed method against the Monte-Carlo method (the benchmark); (II) checking the behavior of the method in case of negligible uncertainties and verifying the consistency with the conventional TTC calculation.

As far as point (I) is concerned, Figure 3 compares the CDF of the TTC calculated with the Monte-Carlo method (with 500 samples) and the probabilistic method, considering stress scenario B and a time horizon of 12 hours. The matching is found to be satisfactory, as confirmed by the comparison of the first two statistical moments (mean and standard deviation) in Table II, while the computational time is extremely lower in the proposed method.

In order to perform validation step (II) for each stress scenario A and B, standard deviations have been set to $1 / 10$ of the above mentioned values for WFs and SPs. Figure 4 reports the CDFs of the TTC for scenario B. 


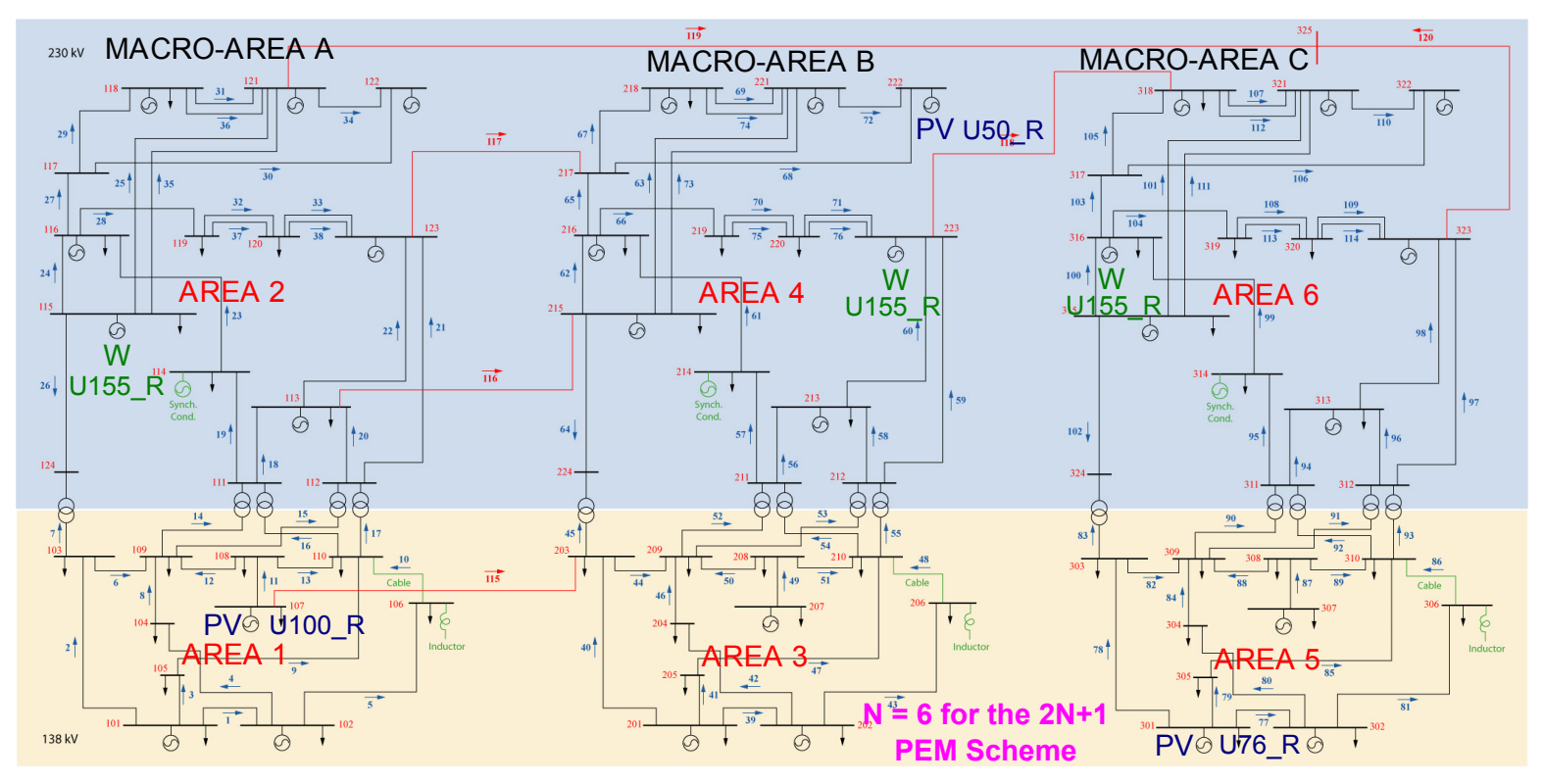

Fig. 2. IEEE RTS-96 network

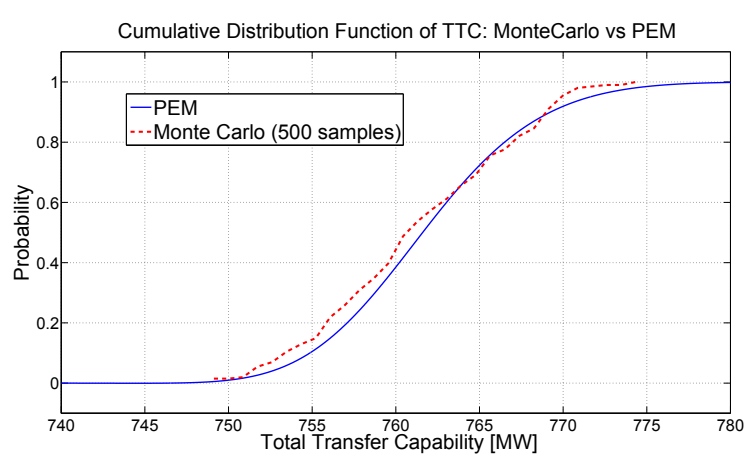

Fig. 3. Comparing the CDF of the TTC from proposed probabilistic method against the benchmark (Montecarlo): validation step I

TABLE II

COMPARISON OF FIRST TWO STATISTICAL MOMENTS OF TTC CDF: MONTE-CARLO VS PROPOSED METHOD

\begin{tabular}{ccc}
\hline & Monte-Carlo (500 samples) & PEM \\
\hline Mean [MW] & 761.6 & 761.9 \\
Standard deviation [MW] & 5.7 & 5.6 \\
Calculation time [minutes] & 240 & 10 \\
\hline
\end{tabular}

It can be noticed that the curve has very little variance and its centered on the TTC value calculated by the conventional method (i.e. $762 \mathrm{MW}$ ).

After that, for each stress scenario (A and B) the goal is to compare the results of the probabilistic calculation with conventional one, accounting for:

- Correlation among the RES injections

- Time horizon of forecast

Table III reports the set of simulation scenarios analyzed.

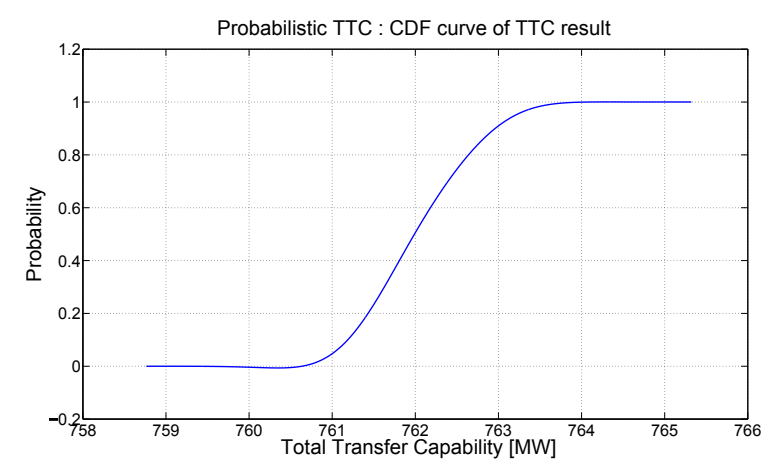

Fig. 4. Assessing the CDF of TTC in case of negligible uncertainties: validation step II

TABLE III

SIMULATION SCENARIOS

\begin{tabular}{rrrrr}
\hline ID & $\begin{array}{r}\text { Area } \\
\text { UP }\end{array}$ & $\begin{array}{r}\text { Area } \\
\text { DOWN }\end{array}$ & $\begin{array}{r}\text { Time } \\
\text { horizon [h] }\end{array}$ & $\begin{array}{r}\text { Distance } \\
\text { among WF's [km] }\end{array}$ \\
\hline A1 & 4 & 6 & 12 & 20 \\
A2 & 4 & 6 & 24 & 20 \\
A3 & 4 & 6 & 12 & 200 \\
A4 & 4 & 6 & 24 & 200 \\
B1 & $3-4$ & $1-2$ & 12 & 20 \\
B2 & $3-4$ & $1-2$ & 24 & 20 \\
B3 & $3-4$ & $1-2$ & 12 & 200 \\
B4 & $3-4$ & $1-2$ & 24 & 200 \\
\hline
\end{tabular}

\section{Effect of linear correlation coefficients among the RES}

Figure 5 compares the CDFs of scenarios A2 and A4 which differ for the distance among WFs, thus also for correlation coefficients among relevant power injections are different $(0.75$ and 0.057 for scenarios A2 and A4). It can be noticed that in case of $\alpha=90 \%$ the NTC value reduces from 363 to 360 MW if the WFs have a higher correlation: this demonstrates that 
the proposed tool is able to quantify the effect of correlation of forecast errors on the final NTC value. These NTC values are lower than TTC calculated via the conventional method, but higher than the NTC estimated using a conservative 5\% TRM.

\section{Effect of time horizon of forecast}

Figure 6 compares the CDFs of the TTC between areas $4+3$ and $1+2$ for scenarios $\mathrm{B} 1$ and $\mathrm{B} 2$ with time horizons equal to 12 and 24 hours. Assuming a value equal to $90 \%$ for $\alpha$ parameter it can be noticed that NTC value passes from 754 to 755 when time horizon passes from 24 to 12 hours.

This slight increase is justified by the modest reduction of the standard deviation of the forecast error of a WT, given by the characteristics in Figure 7: the curve provides the derating factor $\frac{\sigma_{k-\text { hours }}}{\sigma_{24-\text { hours }}}$ estimated from [9] which links the standard deviation $\sigma$ of forecast error for a WT to forecast time horizon. A similar trend holds valid for solar panels [16]. In both cases, the values of NTC are higher than the NTC (724 MW) evaluated via the conventional method, which demonstrates that an accurate evaluation of the forecast uncertainties instead of adopting a prudential margin can lead to a less conservative estimation of the NTC. The same consideration holds valid for A scenarios: given $\alpha=90 \%$ for A1 (12 hours ahead forecasts) and A2 (24 hours ahead forecasts) the NTC values are respectively equal to 364 and $363 \mathrm{MW}$, which are lower than conventional TTC (366 MW) but higher than $348 \mathrm{MW}$ provided by conventional NTC calculation.

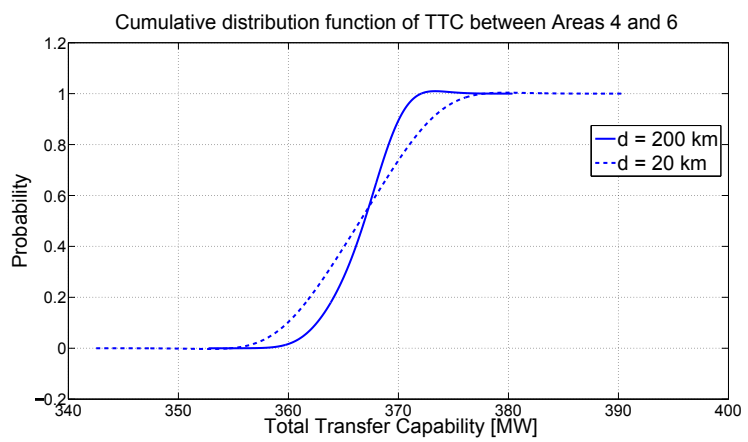

Fig. 5. Comparison of CDFs of TTC between Areas 4 (UP) and 6 (DOWN) for two distances among WFs: $20 \mathrm{~km}$ (dotted line) and $200 \mathrm{~km}$ (solid line)

\section{CONCLUSIONS}

This work proposes a probabilistic procedure based on the innovative coupled implementation of PEM and TPN Transformation. The advantage of the proposed method with respect to the conventional approach is that the reliability margin is not a fixed value (in MW or in \% of the TTC), but it is accurately determined as a function of the uncertainties due to RES forecast errors over the time horizon under study for operational planning purposes. This allows a more precise determination of the transfer capacity allowable for market transactions, avoiding under or over-estimations often brought by the adoption of the conventional method.

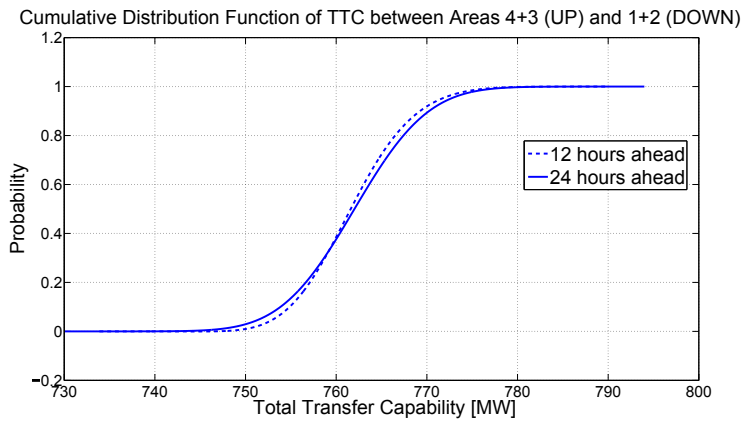

Fig. 6. CDFs of TTC among Areas 3+4 (UP) and 1+2 (DOWN) for two forecast horizons: 12 hours (dotted line) and 24 hours (solid line)

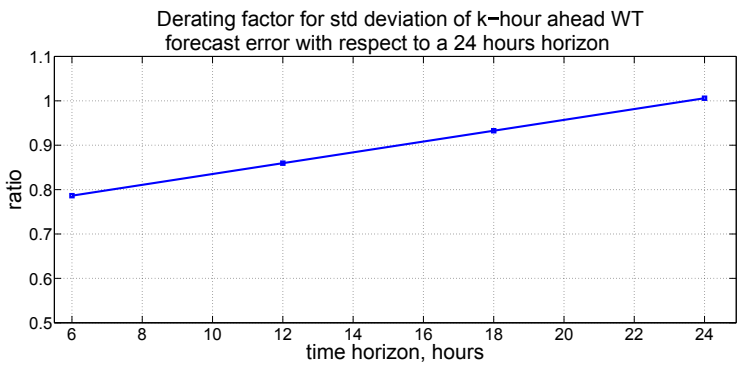

Fig. 7. Derating factor for standard deviation of the forecast error of WTs as a function of time horizon: factor 1 corresponds to a 24 hour time horizon

The proposed approach has been validated against a benchmark method (Monte-Carlo): results show a good matching between the two methods, and significant computational time savings by using the proposed method instead of Monte-Carlo.

\section{APPENDIX}

This Appendix reports the fundamentals of the $2 \mathrm{~N}+1$ PEM Scheme and TPN Transformation which are essential for the proposed probabilistic procedure.

\section{A. $2 N+1$ PEM Scheme}

The $2 N+1$ PEM Scheme is used to calculate the PDF of TTC. The stochastic inputs of this method are the forecast errors on RES (wind and solar injections) and on load absorption, indicated as $X$ with distribution $f_{X}(x)$. The $2 N+1$ PEM Scheme requires to apply the TTC evaluation algorithm to $2 N+1$ different initial network states. This $2 N+1$ starting points are given by replacing the stochastic inputs by:

- 2 vectors for each stochastic variable:

$$
\bar{X}_{l, k}=\left(\mu_{1}, \mu_{2}, \ldots, \bar{x}_{l, k}, \ldots, \mu_{N}\right), k=1,2
$$

where $l$ identify the stochastic variable, $\mu_{i}(i=$ $1, \ldots, N, l \neq i)$ the mean of $i^{\text {th }}$ stochastic input and $\bar{x}_{l, k}$ given by the following formula:

$$
\bar{x}_{l, k}=\mu_{l}+\xi_{l, k} \cdot \sigma_{l}
$$

where $\sigma_{l}$ is the standard deviation of the $l^{\text {th }}$ variable, while $\xi_{l, k}$ is evaluated through the successive relations:

$$
\xi_{l, k}=\frac{\lambda_{l, 3}}{2}+(-1)^{3-k} \cdot \sqrt{\lambda_{l, 4}-\frac{3 \lambda_{l, 3}^{2}}{4}}, k=1,2, \xi_{l, 3}=0
$$


with $\lambda_{l, 3}$ e $\lambda_{l, 4}$ which represent respectively the normalized third and fourth moments, evaluated as a function of the expectation value of an order statistic $E X$ [17]:

$$
\lambda_{l, r}=\frac{1}{r} \cdot \sum_{k=0}^{r-1}(-1)^{k} \cdot\left(\begin{array}{c}
r-1 \\
k
\end{array}\right) \cdot E X_{r-k: r}
$$

where $E X_{r-k: r}$ is determined according to $f_{X}(x)$ and its $\operatorname{CDF} F_{X}(x)$ :

$$
\begin{gathered}
E X_{r-k: r}=\frac{r !}{(j-1) !(r-j) !} \\
\cdot \int_{-\infty}^{+\infty} x \cdot f_{X}(x) \cdot\left[F_{X}(x)\right]^{j-1} \cdot\left[1-F_{X}(x)\right]^{r-j} d x .
\end{gathered}
$$

- 1 vector composed of the mean value $\mu_{i}(i=1, \ldots, N)$ of all the stochastic variables.

For each vector a the TTC evaluation algorithm, presented in section II-B, is run. The results, in terms of generation increase, are stored in the $Z(l, k)$ functions (location $k$ of variable $l$ ). The values of the moments necessary to calculate the CDF of the TTC are obtained according to the following relation:

$$
E\left(Z^{j}\right)=\sum_{l=1}^{N} \sum_{k=1}^{3} w_{l, k} \cdot Z(l, k)^{j}
$$

where the weights $w_{l, k}$ are given by:

$$
\begin{aligned}
& w_{l, k}=\frac{(-1)^{3-k}}{\xi_{l, k} \cdot\left(\xi_{l, 1}-\xi_{l, 2}\right)}, \quad k=1,2, \\
& w_{l, 3}=\frac{1}{N}-\frac{1}{\lambda_{l, 4}-\lambda_{l, 3}^{2}}
\end{aligned}
$$

After the reconstruction of the fundamental moments, the Gram-Charlier [17] method allows to reconstruct the PDF and then the CDF of the TTC.

\section{B. TPN Transformation}

Considering the distribution $f_{X}(x)$ of inputs $X$, the basic idea is to express non normal variables $X=\left(X_{1}, \ldots, X_{N}\right)$ as a third order polynomial of dependent normal variables $W=$ $\left(W_{1}, \ldots, W_{N}\right)$ :

$X_{i}=a_{0, i}+a_{1, i} \cdot W_{i}+a_{2,1} \cdot W_{i}^{2}+a_{3, i} \cdot W_{i}^{3}, \quad i=1, \ldots, N$

where the coefficients $a_{0,1}, a_{1, i}, a_{2, i}, a_{3, i}$ are calculated as functions of $L$-moments:

$$
\begin{aligned}
& a_{0,1}=\lambda_{1, i}-1.81379937 \cdot \lambda_{3, i} \\
& a_{1, i}=2.25518617 \cdot \lambda_{2, i}-3.93740250 \cdot \lambda_{4, i} \\
& a_{2, i}=1.81379937 \cdot \lambda_{3, i} \\
& a_{3, i}=-0.19309293 \cdot \lambda_{2, i}+1.574961 \cdot \lambda_{4, i}
\end{aligned}
$$

Also $W$ variables are dependent and the correlation matrix $R_{W}$ among them can be derived from correlation matrix $R_{X}$ of original variables by solving the equation below for any pair of $X$ variables:

$$
\begin{aligned}
& 6 a_{3, i} a_{3, j} \rho_{W_{i}, W_{j}}^{3}+2 a_{3, i} a_{3, j} \cdot \rho_{W_{i}, W_{j}}^{2}+\left(a_{1, i}+3 a_{3, i}\right) \cdot\left(a_{1, j}+3 a_{3, j}\right) \cdot \\
& \cdot \rho_{W_{i}, W_{j}}+\left[\left(a_{0, i}+a_{2, i}\right) \cdot\left(a_{0, j}+a_{2, j}\right)-\rho_{X_{i}, X_{j}} \cdot \sigma_{i} \cdot \sigma_{j}-\mu_{i} \cdot \mu_{j}\right]=0
\end{aligned}
$$

$R_{W}$ is positive definite matrix and it can be subject to Cholesky decomposition:

$$
R_{W}=G_{W} \cdot G_{W}^{T} .
$$

Using matrix $G_{W}$ it is possible to transform normal dependent variables $W$ into independent normal variable $Y$ to which $2 N+1$ PEM Scheme can be applied. The linear transformation between $W$ and $Y$ is given by:

$$
\begin{aligned}
& Y=G_{W}^{-1} \cdot W \\
& \text { REFERENCES }
\end{aligned}
$$

\section{REFERENCES}

[1] "R\&D Roadmap 2013-2022: Writing history again”, Dec. 2012

[2] D. Cirio, D. Lucarella, S. Massucco, "Online Dynamic Security Assessment to Mitigate the Risk of Blackout in the Italian Power System", European Transactions on Electrical Power (ETEP), Vol.18, No.8, Nov. 2008, pp. 784-801

[3] H. Holttinen et al., "Design and operation of power systems with large amounts of wind power", IEA Wind Task 25, Final report, Phase I, 200608, Helsinki, 2009

[4] S. Alessandrini, S. Sperati, P. Pinson, "The influence of new ECMWF Ensemble Prediction System resolution on wind power forecast accuracy and uncertainty estimation", Adv. Sci. Res., 8, 2012, pp. 143-147

[5] E. Ciapessoni, D. Cirio, A. Pitto, S. Massucco, F. Silvestro, "A Novel Approach to Account for Uncertainty and Correlations in Probabilistic Power Flow", IEEE PES Innovative Smart Grid Technologies (ISGT), Istanbul, 12-15 Oct. 2014

[6] G. Salic, Y. Robours, "Impact of German Wind Generation Forecasts on Net Transfer Capacities", 8th International Conference on the European Energy Market (EEM), Zagreb, 25-27 May 2011

[7] ENTSO-E, "Definition of Transfer Capacities in liberalized Electricity Markets", 2001

[8] ENTSO-E, "Net Transfer Capacities (NTC) and Available Transfer Capacities (ATC) in the Internal Market of Electricity in Europe", 2000

[9] U. Focken, M. Lange, H. P. Waldl, H. G. Beyer, A. Luig "A statistical analysis of the reduction of the wind power prediction error by spatial smoothing effects"', J. Wind Eng. Ind. Aerodyn., Vol. 90, Issue 3, pp. 231-246, 2002

[10] E. Lorenz, J. Hurka, G. Karampela, D. Heinemann, H. Georg Beyer, M. Schneider, "Qualified Forecast of Ensemble Power Production by Spatially Dispersed Gridconnected PV Systems", IEA Task 36, 2007

[11] P.L. Liu, D. Kiureghian, "A Multivariate distribution models with prescribed marginals and covariances", Probab. Eng. Mech., Vol. 1, Issue 2, pp. 105-112, 1986

[12] H. Yang, B. Zou, "The Point Estimate Method using third order polynomial normal transformation technique to solve probabilistic powerflow with correlated wind source and load", 2012 Asia-Pacific Power and Energy Engineering Conference (APPEEC), March 27-29, 2012

[13] E. Rosenblueth, "Point estimates for probability moments", Proc. Nat. Acad. of Sci., Vol 72, Issue 10, pp. 3812-3814, 1975

[14] E. Ciapessoni, D. Cirio, S. Grillo, S. Massucco, A.Pitto, F. Silvestro, "An integrated platform for power system security assessment implementing probabilistic and deterministic methodologies", IEEE Systems Journal, Vol. 7, Issue 4, pp. 845-853, 2013

[15] C. Grigg et al. "The IEEE Reliability Test System-1996. A report prepared by the Reliability Test System Task Force of the Application of Probability Methods Subcommittee", IEEE Trans. on Power System, Vol., no.3, pp. 1010-1020, Aug. 1999

[16] S. Pelland et al. , "Photovoltaic and Solar Forecasting: State of the Art", IEA PVPS Task 14, Report IEA-PVPS T14-01, Oct 2013

[17] J.R.M. Hosking, "L-moments: analysis and estimation of distributions using linear combinations of order statistics", Journal of the Royal Statistical Society, Series B 52, pp. 105-124, 1990 